\section{Einfluß von Thallium auf die Leitfähigkeit von halogendotiertem Selen}

Von H.-P. Hempel, Hans Lauckner und Hedwig Thumann

Laboratorium für Selengleichrichter der Standard Elektrik Lorenz AG, Nürnberg

(Z. Naturforschg. 16 a, 1402-1403 [1961] ; eingeg. am 8. September 1961)

Bei gleichzeitiger Dotierung des Selens mit einem Halogen und Thallium verbinden sich die Zusätze zu einem Halogenid, das praktisch keinen Einfluß auf die elektrische Leitfähigkeit des Selens hat. Diese wird lediglich durch die überschüssige Komponente bestimmt. Ein Thalliumüberschuß von 40 bis $50 \mathrm{~g} / \mathrm{t}$ erhöht den spezifischen Widerstand um 5 bis 6 Größenordnungen. Das Thallium wird sehr wahrscheinlich an Korngrenzen angelagert.

Bekanntlich wird durch Dotieren der obersten Selenschicht mit Thallium die Sperrfähigkeit von Selengleichrichtern verbessert. Es wurde deshalb untersucht, wie sich die Leitfähigkeit von halogendotiertem Selen, wie man es zur Herstellung von Gleichrichtern verwendet, bei Zusatz von Thallium verändert.

\section{Präparation der Proben}

Für die Versuche wurde Boliden-Reinstselen verwendet. Die Spektralanalyse dieses Materials zeigte geringe Spuren von $\mathrm{Cu}, \mathrm{Ca}, \mathrm{Mg}, \mathrm{Fe}, \mathrm{Si}$; jedoch sämtlich unter $1 \mathrm{~g} / \mathrm{t}$. Auch der Gehalt an Chlor, Schwefel und Natrium lag jeweils unter $1 \mathrm{~g} / \mathrm{t}$. Durch Abziehen der beim Einschmelzen des Halogens sich bildenden Häutchen wurde das Material nochmals beträchtlich gereinigt.

Ein Teil des Selens wurde durch Einschmelzen mit $200 \mathrm{~g} / \mathrm{t} \mathrm{Jod}$, der Rest mit $380 \mathrm{~g} / \mathrm{t} \mathrm{Tl} \mathrm{T}_{2}$ Se dotiert. Durch Zusammenschmelzen in verschiedenen Verhältnissen wurden daraus Proben mit gleichzeitiger Jod- und Thalliumdotierung in verschiedenen Molverhältnissen hergestellt. In derselben Weise wurden durch Zusammenschmelzen von Selen mit $150 \mathrm{~g} / \mathrm{t}$ Chlor bzw. $1032 \mathrm{~g} / \mathrm{t}$ $\mathrm{Tl}_{2}$ Se Proben mit gleichzeitiger Chlor- und Thalliumdotierung gewonnen.

Für die Messung der elektrischen Leitfähigkeit wurden Schichten hergestellt. Da beim Aufdampfen von thalliumdotiertem Selen im Vakuum nur ein Teil des zugesetzten $\mathrm{Tl}$ mit verdampft, kam dieses Verfahren nicht in Frage. Statt dessen wurden durch Pressen des erwärmten Selens in einer Stahlschablone zwischen Rein-

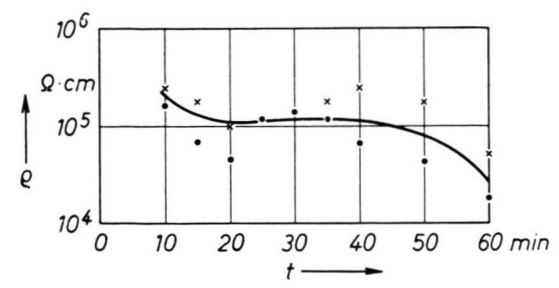

Abb. 1. Abhängigkeit des spezifischen Widerstandes von der Dauer der Temperung $\left(218^{\circ} \mathrm{C}\right)$. Selenprobe mit $8,16 \cdot 10^{-5}$ Atomanteilen Thallium und $7,48 \cdot 10^{-5}$ Atomanteilen Jod. - Graphit-Elektroden, $\times$ Wismut-Elektroden. aluminiumplatten Schichten von $40 \times 10 \times 1 \mathrm{~mm}^{3}$ Größe hergestellt. Diese wurden $30 \mathrm{~min}$ lang bei $218^{\circ} \mathrm{C}$ getempert, um sie völlig in die hexagonal kristalline Form zu überführen. Abb. 1 zeigt, wie sich der spezifische Widerstand einer solchen Schicht beim Tempern verändert.

Die streifenförmigen Schichten wurden an beiden Schmalseiten durch Aufstreichen von Graphitaufschlämmung, z. Tl. auch durch Aufspritzen von flüssigem Wismut, mit Stromkontakten versehen.

\section{Meßmethode}

Für die Widerstandsmessungen wurde bis zu $10^{6} \Omega \mathrm{cm}$ die Sondenmethode benutzt, wobei die Sonden bei jeder Schicht nacheinander an verschiedenen Punkten aufgesetzt wurden. Auch wurden jeweils mehrere Schichten mit gleichem Dotierungsverhältnis hergestellt und gemessen. Die in den Abbildungen angegebenen Widerstandswerte stellen daher die aus durchschnittlich 8 Messungen gewonnenen Mittelwerte dar.

Bei spezifischen Widerständen oberhalb $10^{6} \Omega$ cm versagte die Sondenmethode. Für solche Schichten wurde ein Teraohmmeter benutzt, das den Strom durch die Probe bei bekannter Spannung elektrometrisch als Spannungsabfall an einem Vergleichswiderstand mißt.

\section{Ergebnisse}

Abb. 2 zeigt den Gang des spezifischen Widerstandes mit zunehmender Thallium- und abnehmender Chlordotierung. Der Widerstand nimmt allmählich zu und

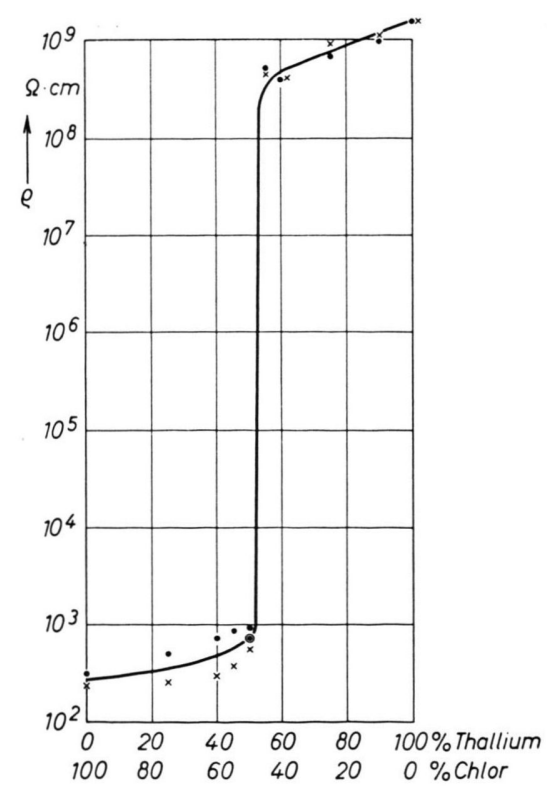

Abb. 2. Spezifischer Widerstand des Selens in Abhängigkeit vom Verhältnis der Thallium- zur Chlordotierung. @ GraphitElektroden, $\times$ Wismut-Elektroden, $\odot$ spezifischer Widerstand des undotierten Selens. 
erreicht bei gleicher molarer Dotierung mit beiden Substanzen fast genau den Wert des undotierten AusgangsSelens, der zum Vergleich an dieser Stelle eingetragen ist. Bei weiterem Thalliumüberschuß steigt die Kurve steil um 6 Größenordnungen an, um bei etwa $10 \%$ molarem Überschuß an $\mathrm{Tl}_{2} \mathrm{Se}$ wieder in einen gemäßigten Anstieg überzugehen.

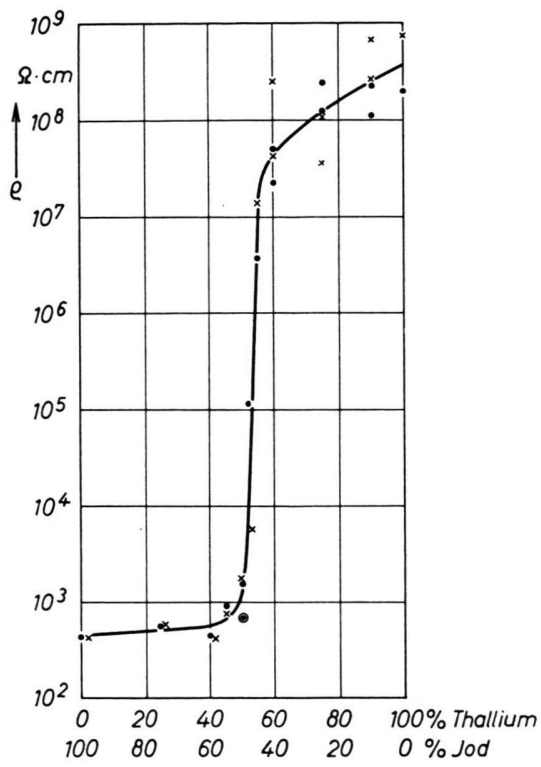

Abb. 3. Spezifischer Widerstand des Selens in Abhängigkeit vom Verhältnis der Thallium- zur Joddotierung. Bezeichnungen wie in Abb. 2.

Das gleiche Bild zeigt Abb. 3 für Jod- und Thalliumdotierung. Wegen der 2,7-mal geringeren molaren Ausgangskonzentration der Dotierungssubstanzen ist der spezifische Widerstand von vornherein etwas höher und der Anstieg nicht so steil. Hier konnten im Bereich des steilen Anstiegs einige Zwischenwerte ermittelt werden.

\section{Diskussion}

Daß bei gleicher molarer Dotierung mit Thallium und Halogen der Widerstand des kristallisierten Selens gleich dem des undotierten Selens ist, läßt sich zwanglos dadurch erklären, daß sich beim Zusammenschmel-

1 H. W. Henkels u. J. Maczuk, J. Appl. Phys. 25, 1 [1954]. zen Thallium und Halogen quantitativ verbinden - was sich auch chemisch nachweisen läßt - und daß das Thalliumhalogenid die Leitfähigkeit des Selens nicht merklich beeinflußt. Der spezifische Widerstand des Selens wird also nur durch die überschüssige Komponente bestimmt. Überschüssiges Thallium erhöht ihn um mehrere Größenordnungen, wie Abb. 4 zeigt.

Nach Messungen von Henkels und Maczuk ${ }^{1}$ ist der Wechselstromwiderstand einer mit $193 \mathrm{~g} / \mathrm{t}$ Thallium dotierten Selenprobe $10^{4}$ - bis $10^{6}$-mal (je nach den Kristallisationsbedingungen) geringer als ihr Gleichstromwiderstand. Daraus kann man folgern, daß das Thallium sich an den Korngrenzen anlagert und deren Leit-

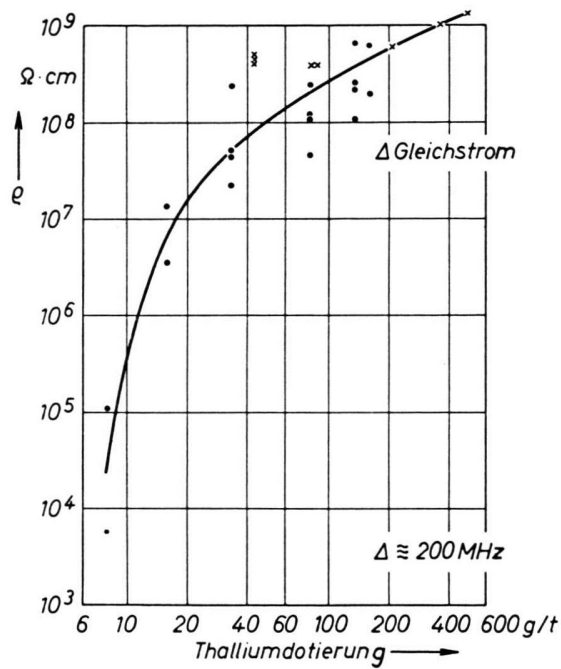

Abb. 4. Spezifischer Widerstand des Selens in Abhängigkeit vom Thalliumüberschuß. $\times$ Meßpunkte aus Abb. 2, Meßpunkte aus Abb. 3, $\triangle$ Meßwerte nach Henkels und Maczur ${ }^{1}$.

fähigkeit stark verringert, während die Leitfähigkeit der Kristallite praktisch unverändert bleibt. Diese Auffassung wird durch Untersuchungen von GudDen und Lenovec $^{2}$ gestützt. Diese Autoren beobachten eine ungewöhnlich rasche Verschiebung der Grenze zwischen einem gutleitenden und einem infolge Thalliumdotierung schlechtleitenden Bereich eines Selenstäbchens durch Diffusion von Thalliumionen im elektrischen Feld. Diese Diffusion findet zweifellos entlang den Korngrenzen statt.

2 B. Gudden u. K. Lehovec, Z. Naturforschg. 1, 508 [1946].

\section{BERICHTIGUNG}

Zu R. Rodenberg, Note on the Introduction of Form Factors in the Theory of (e, $\mathscr{N})$-processes, Band 16 a, 1243 [1961].

Auf S. 1243, rechte Spalte, muß die 2. Zeile von oben lauten:

given in (I. 13), both act together in the cross section

Nachdruck - auch auszugsweise - nur mit schriftlicher Genehmigung des Verlags gestattet 